JOURNAL OF INTEGRAL EQUATIONS

AND APPLICATIONS

Volume 16, Number 1, Spring 2004

\title{
A NOTE ON THE FREDHOLM PROPERTY OF PARTIAL INTEGRAL EQUATIONS OF ROMANOVSKIJ TYPE
}

\author{
J. APPELL, I.A. ELETSKIKH AND A.S. KALITVIN
}

ABSTRACT. Some conditions are given, both necessary and sufficient, under which a partial integral equation of Romanovskij type defines a Fredholm operator of index zero in the space of continuous functions.

In 1932, Romanovskij [6] has described a problem in the theory of Markov chains with two-sided link which leads to an equation of the form

$$
x(t, s)=R x(t, s)+f(t, s), \quad(t, s) \in D:=[a, b] \times[a, b],
$$

where $R$ is the linear operator defined by

$$
R x(t, s)=\int_{a}^{b} m(t, s, \sigma) x(\sigma, t) d \sigma
$$

which contains some continuous or measurable kernel function $m$ : $D \times[a, b] \rightarrow \mathbf{R}$. A particular feature of the operator (2) is that first the two variables in the unknown integrand $x$ are inverted, and afterwards the integration is carried out with respect to the first variable.

Equation (1) has been studied for continuous kernel functions in [6] by means of Fredholm determinants. In this connection it turned out that many results on the problem (1) are quite different from classical results on Fredholm integral equations, mainly due to the fact that the operator (2) is not compact and not even an integral operator. It is natural (and now common sense) to call operators of the form (2) partial integral operators, inasmuch as the integration in (2) is carried out only with respect to one variable while the other variables are "frozen."

AMS Mathematics Subject Classification. 45A05, 45B05, 45P05, 46E15, 47A53, 47B38, 47G10.

Key words and phrases. Partial integral operator, partial integral equation, Romanovskij type equation, Fredholm operator.

Received by the editors on August 11, 2003.

Copyright (C)2004 Rocky Mountain Mathematics Consortium 
If one writes the integral in (2) without switching arguments, i.e.,

$$
M x(t, s)=\int_{a}^{b} m(t, s, \sigma) x(t, \sigma) d \sigma
$$

one gets an operator with completely different properties. So, in contrast to the operator (3), the square $R^{2}$ of the operator (2) is compact in the Chebyshev space $C(D)$ of all continuous real functions on $D$, or in the Lebesgue space $L_{p}(D)$ of all $p$-summable, $1 \leq p<$ $\infty$, respectively essentially bounded, $p=\infty$, real functions on $D$. Consequently, the operator $I-R$ is Fredholm in these spaces, while the operator $I-M$ is not Fredholm even in the most trivial case of a constant kernel function $m$.

Equation (1) may be considered as a special case of the more general equation of Romanovskij type

$$
x=K \Pi x+f,
$$

where $\Pi$ denotes the "argument switch" operator $\Pi x(t, s)=x(s, t)$ and the operator $K$ is of the form $K=L+M+N$ with $M$ as in (3),

$$
L x(t, s)=\int_{a}^{b} l(t, s, \tau) x(\tau, s) d \tau,
$$

and

$$
N x(t, s)=\int_{a}^{b} \int_{a}^{b} n(t, s, \tau, \sigma) x(\tau, \sigma) d \sigma d \tau .
$$

Observe that (5) is again a partial integral operator, while (6) is a usual integral operator of Fredholm type on functions of two variables. In what follows we assume that the kernel functions $l, m$ and $n$ are measurable on their domains.

Various analytical and topological properties of the operators (3), (5) and (6) have been studied in the monograph [3]. Moreover, in the thesis [2] one may find criteria for the operator equation

$$
x=K x+f
$$


to be Fredholm in the spaces $C(D)$ (which means that the operator $I-K$ is Fredholm). Surprisingly, such criteria are not only of theoretical interest but also quite useful in view of applications [1]. On the other hand, Fredholm criteria are not known, even in the case of continuous kernel functions, for the operator equation (4) and related equations, and it is the aim of this short note to provide such criteria.

To treat this problem more systematically, we consider the most general equation of Romanovskij type

$$
x=K_{i} x+f, \quad i=1,2,3,4,
$$

where we have used the shortcuts

$$
\begin{cases}K_{1}=L \Pi+M+N, & K_{2}=L \Pi+M+N \Pi, \\ K_{3}=L+M \Pi+N, & K_{4}=L+M \Pi+N \Pi .\end{cases}
$$

We show now how to obtain Fredholm conditions for the Romanovskij equation (8) under some natural additional hypotheses on the kernel functions involved.

Recall that a measurable function $m: D \times[a, b] \rightarrow \mathbf{R}$ is called $L_{1}$-continuous if for each $\varepsilon>0$ one may find $\delta>0$ such that $\left|t-t^{\prime}\right|<\delta$ and $\left|s-s^{\prime}\right|<\delta$ implies that

$$
\int_{a}^{b}\left|m(t, s, \sigma)-m\left(t^{\prime}, s^{\prime}, \sigma\right)\right| d \sigma<\varepsilon,
$$

and $L_{1}$-bounded if

$$
C(m):=\sup _{(t, s) \in D} \int_{a}^{b}|m(t, s, \sigma)| d \sigma<\infty .
$$

The corresponding properties of the kernel functions $l$ and $n$ are defined similarly. Clearly, any continuous kernel function is both $L_{1}$-continuous and $L_{1}$-bounded.

Recall (see, e.g., [4]) that a bounded linear operator is Fredholm of index zero, i.e., has finite-dimensional kernel and cokernel of the same dimension, if and only if it may be represented as a sum of an invertible and a compact operator. 
Theorem 1. Suppose that the kernel functions $l, m$ and $n$ are $L_{1}$-continuous and $L_{1}$-bounded on their domains. Then equation (8) is Fredholm of index zero in $C(D)$ for $i=1,2$ if and only if the operator $I-M$ is Fredholm of index zero in $C(D)$. Similarly, equation (8) is Fredholm of index zero in $C(D)$ for $i=3,4$ if and only if the operator $I-L$ is Fredholm of index zero in $C(D)$.

Proof. Consider the case $i=1$, i.e., the equation

$$
x=(L \Pi+M+N) x+f .
$$

We may rewrite this equation in the form

$$
(I-M)(I-L \Pi) x=(N+M L \Pi) x+f .
$$

By our assumption on the kernel functions $l, m$ and $n$, the operator $N$ from (6) is compact in $C(D)$, see, e.g., [7]. Moreover, the operator $M L \Pi$ may be represented in the form

$$
M L \Pi x(t, s)=\int_{a}^{b} \int_{a}^{b} m(t, s, \tau) l(t, \tau, \sigma) x(\tau, \sigma) d \sigma d \tau
$$

by Fubini's theorem. From the estimate

$$
\begin{aligned}
\int_{a}^{b} \int_{a}^{b}|m(t, s, \tau) l(t, \tau, \sigma)| d \sigma d \tau & \leq \int_{a}^{b}|m(t, s, \tau)|\left(\int_{a}^{b}|l(t, \tau, \sigma)| d \sigma\right) d \tau \\
& \leq C(m) C(l)
\end{aligned}
$$

it follows that the kernel function $(t, s, \tau, \sigma) \mapsto m(t, s, \tau) l(t, \tau, \sigma)$ is $L_{1}$-bounded. Let $\varepsilon>0$. Since the kernel functions $l$ and $m$ are $L_{1}$-continuous, we may find $\delta>0$ such that

$$
\begin{gathered}
\int_{a}^{b}\left|l(t, s, \tau)-l\left(t^{\prime}, s^{\prime}, \tau\right)\right| d \tau<\frac{\varepsilon}{2 C(m)}, \\
\int_{a}^{b}\left|m(t, s, \sigma)-m\left(t^{\prime}, s^{\prime}, \sigma\right)\right| d \sigma<\frac{\varepsilon}{2 C(l)} .
\end{gathered}
$$


Consequently,

$$
\begin{aligned}
\int_{a}^{b} \int_{a}^{b}\left|m(t, s, \tau) l(t, \tau, \sigma)-m\left(t^{\prime}, s^{\prime}, \tau\right) l\left(t^{\prime}, \tau, \sigma\right)\right| d \sigma d \tau \\
\leq \int_{a}^{b} \int_{a}^{b}\left|m(t, s, \tau)-m\left(t^{\prime}, s^{\prime}, \tau\right)\right||l(t, \tau, \sigma)| d \sigma d \tau \\
\quad+\int_{a}^{b} \int_{a}^{b}\left|m\left(t^{\prime}, s^{\prime}, \tau\right)\right|\left|l(t, \tau, \sigma)-l\left(t^{\prime}, \tau, \sigma\right)\right| d \sigma d \tau \\
<C(l) \frac{\varepsilon}{2 C(l)}+C(m) \frac{\varepsilon}{2 C(m)}=\varepsilon .
\end{aligned}
$$

We conclude that the kernel function $(t, s, \tau, \sigma) \mapsto m(t, s, \tau) l(t, \tau, \sigma)$ is also $L_{1}$-continuous, and so the corresponding operator (11) is compact in the space $C(D)$. Now, since the Fredholm property is invariant under compact perturbations (see, e.g., [5]) equation (10) is Fredholm of index zero if and only if the reduced equation

$$
(I-M)(I-L \Pi) x=f
$$

is Fredholm of index zero. Again, from Fubini's theorem, it follows that the square of the operator $L \Pi$ may be represented in the form

$$
(L \Pi)^{2} x(t, s)=\int_{a}^{b} \int_{a}^{b} l(t, s, \tau) l(s, \tau, \xi) x(\tau, \xi) d \xi d \tau .
$$

As above, one may show that the kernel function

$$
(t, s, \tau, \xi) \mapsto l(t, s, \tau) l(s, \tau, \xi)
$$

of the operator (13) is both $L_{1}$-bounded and $L_{1}$-continuous, and so this operator is compact in $C(D)$. It follows that $I-L \Pi$ is Fredholm of index zero in $C(D)$ and hence may be represented as sum $I-L \Pi=J+Q$ of an invertible operator $J$ and a compact operator $Q$. With this notation, equation (12) reads

$$
(I-M) J x+(I-M) Q x=f .
$$

Since $(I-M) Q$ is compact in $C(D)$, equation (12) is Fredholm if and only if the equation

$$
(I-M) J x=f
$$


is Fredholm, and this is certainly equivalent to the fact that the operator $I-M$ is Fredholm of index zero. So the assertion is proved for the operator $K_{1}$. The proof for the other three operators in (9) is similar.

In order to apply Theorem 1, one may use various conditions from [2] which guarantee the Fredholm property of $I-M$ or $I-L$ and are formulated in terms of the generating kernel functions $m$ or $l$, respectively. We give such a condition for $I-M$ in the special case of a degenerate kernel function.

To this end, suppose that the kernel function $m$ in (3) has the form

$$
m(t, s, \sigma)=m_{0}(t, s, \sigma)+\sum_{j=1}^{k} m_{j}(t, s) \hat{m}_{j}(\sigma),
$$

where the system $\left\{m_{1}(t, s), \ldots, m_{k}(t, s)\right\}$ is linearly independent, the functions from the system $\left\{\hat{m}_{1}(\sigma), \ldots, \hat{m}_{k}(\sigma)\right\}$ are mutually orthogonal, and

$$
\max _{(t, s) \in D} \int_{a}^{b}\left|m_{0}(t, s, \sigma)\right| d \sigma<1 .
$$

Putting this kernel function into (3), the equation $x=M x+f$ becomes

$$
x=P x+g
$$

with

$$
\begin{aligned}
P x(t, s) & =\sum_{j=1}^{k} \int_{a}^{b} p_{j}(t, s) \hat{m}_{j}(\sigma) x(t, \sigma) d \sigma, \\
g(t, s) & =f(t, s)+\int_{a}^{b} r(t, s, \sigma) f(t, \sigma) d \sigma,
\end{aligned}
$$

where we have put

$$
p_{j}(t, s)=m_{j}(t, s)+\int_{a}^{b} r(t, s, \sigma) m_{j}(t, \sigma) d \sigma, \quad j=1,2, \ldots, k
$$

and

$$
r(t, s, \sigma)=\sum_{j=1}^{\infty} m_{0}^{(j)}(t, s, \sigma)
$$


with $m_{0}^{(j)}$ defined iteratively by $m_{0}^{(1)}(t, s, \sigma)=m_{0}(t, s, \sigma)$ and

$$
m_{0}^{(j)}(t, s, \sigma)=\int_{a}^{b} m_{0}^{(j-1)}(t, s, \tau) m_{0}(t, \tau, \sigma) d \tau, \quad j=2,3, \ldots
$$

For $j=1,2, \ldots, k$, we introduce the functions of one variable

$$
x_{j}(t)=\int_{a}^{b} \hat{m}_{j}(\sigma) x(t, \sigma) d \sigma, \quad g_{j}(t)=\int_{a}^{b} \hat{m}_{j}(\sigma) g(t, \sigma) d \sigma,
$$

and

$$
\nu_{i j}(t)=\int_{a}^{b} \hat{m}_{i}(\sigma) p_{j}(t, \sigma) d \sigma, \quad i, j=1,2, \ldots, k .
$$

Then (16) may be equivalently written as system

$$
x_{i}(t)-\sum_{j=1}^{k} \nu_{i j}(t) x_{j}(t)=g_{i}(t), \quad i=1,2, \ldots, k,
$$

and the operator $I-M$ is Fredholm of index zero if and only if the determinant function

$$
D(t)=\operatorname{det}\left(\delta_{i j}-\nu_{i j}(t)\right)=\left|\begin{array}{cccc}
1-\nu_{11}(t) & -\nu_{12}(t) & \cdots & -\nu_{1 k}(t) \\
-\nu_{21}(t) & 1-\nu_{22}(t) & \cdots & -\nu_{2 k}(t) \\
\cdot & \cdot & \cdots & \cdot \\
\cdot & \cdot & \cdots & \cdot \\
\cdot & \cdot & \cdots & \cdot \\
\cdot & \cdot & \cdots & \cdot \\
-\nu_{k 1}(t) & -\nu_{k 2}(t) & \cdots & 1-\nu_{k k}(t)
\end{array}\right|
$$

is different from zero, see [2]. We summarize with the following

Theorem 2. Suppose that the kernel functions $l, m$ and $n$ are $L_{1}$-continuous and $L_{1}$-bounded on their domains. Then the operators $I-K_{1}=I-L \Pi-M-N$ and $I-K_{2}=I-L \Pi-M-N \Pi$ are Fredholm of index zero in $C(D)$ if and only if the determinant function (20) is different from zero for $a \leq t \leq b$, where the functions $\nu_{i j}(t)$ are given by (18). 
Of course, an analogous result holds for the operators $I-K_{3}=$ $I-L-M \Pi-N$ and $I-K_{4}=I-L-M \Pi-N \Pi$ if the kernel function $l$ in (5) has the special degenerate form

$$
l(t, s, \tau)=l_{0}(t, s, \tau)+\sum_{j=1}^{k} l_{j}(t, s) \hat{l}_{j}(\tau) .
$$

\section{REFERENCES}

1. J. Appell, A.S. Kalitvin and Z.M. Nashed, On some partial integral equations arising in the mechanics of solids, Z. Angew. Math. Mech. 79 (1999), 703-713.

2. E.V. Frolova, Linear operators with partial integrals in spaces of continuous functions, Ph.D. thesis, University of Lipetsk, 2000 (in Russian).

3. A.S. Kalitvin, Linear operators with partial integrals, Voronezh, 2000 (in Russian).

4. L.V. Kantorovich and G.P. Akilov, Functional analysis, Nauka, Moscow, 1977; English transl.: Pergamon Press, Oxford, 1982.

5. T. Kato, Perturbation theory for linear operators, Springer, New York, 1966.

6. V.I. Romanovskij, Sur une classe d'équations intégrales linéaires, Acta Math. 59 (1932), 99-208.

7. P.P. Zabrejko, A.I. Koshelev, M.A. Krasnosel'skij, S.G. Mikhlin, L.S. Rakovshchik and V.Ya. Stetsenko, Integral equations - A reference text, Nauka, Moscow, 1968; English transl.: Noordhoff, Leyden, 1975.

University of Würzburg, Department of Mathematics, Am Hubland, D-97074 Würzburg, Germany

E-mail address: appell@mathematik.uni-wuerzburg.de

Eletskij State University, Department of Mathematics, Ul. Lenina 91, R-399770 Elets, Russian FedERATION

E-mail address: elpinst@yelets.lipetsk.ru

Lipetsk Pedagogical State University, Department of Mathematics, Ul. Lenina 42, R-398020 Lipetsk, Russian Federation

E-mail address: kalitvin@lipetsk.ru 